\title{
To an Axiomatic Model of Rate of Growth
}

\author{
Václav Studený ${ }^{1}$, Ivan Mezník ${ }^{2}$ \\ ${ }^{1}$ Department of Applied Mathematics, Faculty of Economics, and Administration, Masaryk University, Brno, Czech Republic \\ ${ }^{2}$ Institute of Informatics, Faculty of Business and Management, Brno University of Technology, Brno, Czech Republic \\ Email:Vclv.St@gmail.com, meznik@fbm.vutbr.cz
}

Received July 7, 2012; revised January 6, 2013; accepted January 13, 2013

Copyright (C) 2013 Václav Studený, Ivan Mezniík. This is an open access article distributed under the Creative Commons Attribution License, which permits unrestricted use, distribution, and reproduction in any medium, provided the original work is properly cited.

\begin{abstract}
In the paper an axiomatic approach to express rates of growth is presented. The formula is given of rate of growth at a point as the limit case of rate of growth on an interval and the inverse formula is derived to compute present and future value of capital for an integrable rate of growth. Incidentally some inconsistencies in currently used formulas are pointed out.
\end{abstract}

Keywords: Interest Rate; Inflation Rate; Rate of Growth

\section{Introduction}

The concept of an average change of an objective function plays a crucial role in financial mathematics. Reflecting the objective function $f$, it is called an interest rate, an inflation rate, and so on. It is given as the value of

$$
(f(t+1)-f(t)) / f(t) .
$$

For a steady state function the same result may be obtained from the formula

$$
\left(\frac{(f(t+\delta)-f(t))}{f(t)}\right)^{\left(\frac{1}{\delta}\right)} .
$$

In macroeconomics a similar, but instantaneous measure, related to a point is needed. Baro (2003) employs the formula $f^{\prime}(t) / f(t)$ (see [1]) which is in fact an average change of the first derivative. The relation between an average change on an interval and an average change of its derivative has not been tackled in the literature. This leads to the problem of

$\lim _{\delta \rightarrow 0}((f(t+\delta)-f(t)) / f(t))^{\left(\frac{1}{\delta}\right)}$. Further, it is desirable to find a formula that gives the future value of the objective function including the case when the rate of growth is neither constant nor piecewise constant function. For a constant rate of growth function with values $\xi$ we have the formula $f(t)=f(0) \cdot(1+\xi)^{t}$, but its generalization $f(0) \cdot \mathrm{e}^{\int_{0}^{x} \xi(s) \mathrm{d} s}$ (see [2] among others) does not work, because the substitution of constant function with value $\xi(t)=\bar{\xi}$ does not yield $f(t)=f(0) \cdot(1+\bar{\xi})^{t}$. Accordingly the aims of the paper are as follows.

1) To define the concept of a rate of change by means of axioms (Section 2).

2) To formulate the notion of a steady state function to model existing interest rates and to find corresponding computation formulas (Section 3).

3) To derive a limit version of a rate of growth (Section 4).

4) To find the inverse formula that enables to calculate the values of a state function (Sections 5 and 6).

5) To point out to some impacts on currently used formulas in financial mathematics (Section 7).

\section{Axioms}

The symbol $\mathbb{R}$ denotes the set of real numbers. Consider a quantity attaining values $y_{1}, y_{2} \in \mathbb{R}$ for $x_{1}$, $x_{2} \in \mathbb{R}$ respectively. A function $\kappa: \mathbb{R}^{4} \rightarrow \mathbb{R}$ is said to be a generalized rate of growth function (shortly rate of growth function) if the following Axioms A1-A4 are satisfied:

Axiom A1.

$$
\kappa\left(x_{1}, y_{1}, x_{2}, y_{2}\right)=\kappa\left(x_{1}+t, y_{1}, x_{2}+t, y_{2}\right)
$$

for any $t \in \mathbb{R}$ (invariance with respect to shift of time).

\section{Axiom A2.}

$$
\kappa\left(x_{1}, y_{1}, x_{2}, y_{2}\right)=\kappa\left(x_{1}, y_{1} \cdot k, x_{2}, y_{2} \cdot k\right)
$$

for any $k \in \mathbb{R}$ (invariance with respect to homoteties).

Axiom A3. $\kappa$ is increasing with respect to the first and fourth variables and decreasing with respect to the 
second and third variables.

Axiom A4.

$$
\kappa\left(x_{1}, y, x_{2}, y\right)=0
$$

for any $y \in \mathbb{R}$ (initial condition- $\kappa$ has zero value for constant functions).

\section{Steady State Functions}

\subsection{Definition}

Let $\kappa$ be a rate of growth function. For a function $f: \mathbb{R} \rightarrow \mathbb{R}$ the function

$$
F_{f}:\left(x_{1}, x_{2}\right) \mapsto \kappa\left(x_{1}, f\left(x_{1}\right), x_{2}, f\left(x_{2}\right)\right)
$$

is called a $\kappa$-rate of growth of $f$ related to $\left\langle x_{1}, x_{2}\right\rangle$. A function $f$ is called a $\kappa$-steady state function if $F_{f}$ is a constant function. For the simplicity we omit $\kappa$ if it is clear from the context. Verbally, $F_{f}$ does not depend on the choice $x_{1}, x_{2}$.

\subsection{Lemma}

1) Every constant function is a $\boldsymbol{K}$-steady state function for any rate of growth function $\kappa$.

2) Let $\kappa$ be rate of growth function. Then there exists a function $f: \mathbb{R}^{2} \rightarrow \mathbb{R}$ such that

$$
\kappa\left(x_{1}, y_{1}, x_{2}, y_{2}\right)=\lambda\left(x_{2}-x_{1}, \frac{y_{2}}{y_{1}}\right)
$$

which is decreasing with respect to the first variable, increasing with respect to the second variable and it holds $\lambda(x, 0) \equiv 0$.

Proof: The statement 1) follows immediately from Axiom A4. Now, by Axiom 1

$$
\kappa\left(x_{1}, y_{1}, x_{2}, y_{2}\right)=\kappa\left(0, y_{1}, x_{2}-x_{1}, y_{2}\right)
$$

and by Axiom 2

$$
\kappa\left(0, y_{1}, x_{2}-x_{1}, y_{2}\right)=\kappa\left(0,1, x_{2}-x_{1}, \frac{y_{2}}{y_{1}}\right) .
$$

Putting $\lambda\left(x_{2}-x_{1}, \frac{y_{2}}{y_{1}}\right)=\kappa\left(0,1, x_{2}-x_{1}, \frac{y_{2}}{y_{1}}\right)$ we get (1). The properties of $\lambda$ are obvious and hence the statemnt 2) holds true.

\subsection{Theorem}

Let $f: \mathbb{R} \rightarrow \mathbb{R}$ be a continuous $\kappa$-steady state function. Then $f$ is an exponential function, i.e. $f: x \mapsto A \mathrm{e}^{B x}$ for some constants $A$ a $B$.

Proof: Let $x_{1}$ a $\quad x_{2}=x_{1}+h$ be given. Then there holds

$$
\begin{aligned}
& \kappa\left(x_{2}, f\left(x_{2}\right), x_{2}+h, f\left(x_{2}+h\right)\right) \\
& =\kappa\left(x_{1}+h, f\left(x_{1}+h\right), x_{1}+2 h, f\left(x_{1}+2 h\right)\right)
\end{aligned}
$$

Since $f$ is a $\kappa$-steady state function, from Definition 3.1 it follows

$$
\begin{aligned}
& \kappa\left(x_{2}, f\left(x_{2}\right), x_{2}+h, f\left(x_{2}+h\right)\right) \\
& =\kappa\left(x_{1}, f\left(x_{1}\right), x_{1}+h, f\left(x_{1}+h\right)\right)
\end{aligned}
$$

and with a view to (1) we get

$$
\lambda\left(h, \frac{f\left(x_{1}+2 h\right)}{f\left(x_{1}+h\right)}\right)=\lambda\left(h, \frac{f\left(x_{1}+h\right)}{f\left(x_{1}\right)}\right) .
$$

As $\lambda$ is injective in any variable, it holds

$$
\frac{f\left(x_{1}+2 h\right)}{f\left(x_{1}+h\right)}=\frac{f\left(x_{2}+h\right)}{f\left(x_{2}\right)}=\frac{f\left(x_{1}+h\right)}{f\left(x_{1}\right)}
$$

and hence

$$
f\left(x_{1}+2 h\right)=\frac{\left(f\left(x_{1}+h\right)\right)^{2}}{f\left(x_{1}\right)} .
$$

Further, by induction

$$
\begin{aligned}
f\left(x_{1}+n h\right) & =\frac{f\left(x_{1}+h\right)}{f\left(x_{1}\right)} \cdot f\left(x_{1}+(n-1) h\right) \\
& =\left(\frac{f\left(x_{1}+h\right)}{f\left(x_{1}\right)}\right)^{n} .
\end{aligned}
$$

From here it follows that the values of $f$ at all equidistant points form a geometric sequence. Moreover, the implication

$$
\begin{aligned}
\frac{f\left(x_{1}+h / 2\right)}{f\left(x_{1}\right)} & =\frac{f\left(x_{1}+h\right)}{f\left(x_{1}+h / 2\right)} \Rightarrow \frac{f\left(x_{1}+h\right)}{f\left(x_{1}\right)} \\
& =\left(\frac{f\left(x_{1}+h / 2\right)}{f\left(x_{1}\right)}\right)^{2}
\end{aligned}
$$

holds true. Therefore $f$ attains the values of some exponential function at all points of the set $\left\{\frac{a h}{2^{b}}, a \in \mathbb{N}, b \in \mathbb{N}\right\}$. Since this set is dense in $\mathbb{R}$, the proof is completed because of we obtained

$$
f(x)=f\left(x_{1}\right)\left(\frac{f\left(x_{1}+h\right)}{f\left(x_{1}\right)}\right)^{-\frac{x_{1}-x}{h}}
$$

for all $x \in \mathbb{R}$ and coosing for instance $x_{1}=0, h=1$ we obtain

$$
f(x)=f(0) \cdot \mathrm{e}^{\ln (f(1) / f(0)) \cdot x}
$$

so $f: x \mapsto A \mathrm{e}^{B x}$, where $A=f(0), B=(f(1))-(f(0))$. (For more details about solving functional equations see 
[3]).

\subsection{Theorem}

Let an exponential function $f: x \mapsto A \mathrm{e}^{B x}$ be a $\kappa$-steady state function. Then there exists an increasing function $\phi$ with the property

$$
\kappa\left(x_{1}, y_{1}, x_{2}, y_{2}\right)=\phi\left(\left(\frac{y_{2}}{y_{1}}\right)^{\frac{1}{x_{2}-x_{1}}}\right) .
$$

Proof: From the assumption for $f$ it follows that there holds

$$
\kappa\left(x_{1}, A \mathrm{e}^{B x_{1}}, x_{2}, A \mathrm{e}^{B x_{2}}\right)=\kappa\left(x_{3}, A \mathrm{e}^{B x_{3}}, x_{4}, A \mathrm{e}^{B x_{4}}\right)
$$

for all $x_{1}<x_{2}, x_{3}<x_{4}$. Denoting $h=x_{2}-x_{1}$ we get (in a view of (1))

$$
\begin{aligned}
& \kappa\left(x_{1}, A \mathrm{e}^{B x_{1}}, x_{2}, A \mathrm{e}^{B x_{2}}\right) \\
& =\lambda\left(x_{2}-x_{1}, \frac{A \mathrm{e}^{B x_{2}}}{A \mathrm{e}^{B x_{1}}}\right)=\lambda\left(h, \mathrm{e}^{B h}\right)=\mathrm{const}
\end{aligned}
$$

for all $B$. Further, putting $B=\ln \left(z^{1 / h}\right)$ and using (1) we get

$$
\lambda(h, z)=\lambda\left(h, \mathrm{e}^{h \ln \left(z^{1 / h}\right)}\right)=\lambda\left(1, \mathrm{e}^{\ln \left(z^{1 / h}\right)}\right)=\lambda\left(1, z^{\frac{1}{h}}\right)
$$

and

$$
\begin{aligned}
\kappa\left(x_{1}, y_{1}, x_{2}, y_{2}\right) & =\kappa\left(0,1,1,\left(\frac{y_{2}}{y_{1}}\right)^{\frac{1}{x_{2}-x_{1}}}\right) \\
& =\phi\left(\left(\frac{y_{2}}{y_{1}}\right)^{\frac{1}{x_{2}-x_{1}}}\right)
\end{aligned}
$$

as required.

\subsection{Note}

In financial mathematics the translation

$$
\phi: x \mapsto x-1
$$

is employed and consequently the rate of growth function is of the form

$$
\kappa_{1}\left(x_{1}, y_{1}, x_{2}, y_{2}\right)=\left(\frac{y_{2}}{y_{1}}\right)^{\frac{1}{x_{2}-x_{1}}}-1
$$

which is called a a compound interest (per unit of time). Besides (more or less from historical reasons) also a simple interest (per unit of time) is used, given by

$$
\hat{\kappa}\left(x_{1}, y_{1}, x_{2}, y_{2}\right)=\frac{y_{2}-y_{1}}{y_{0} \cdot\left(x_{2}-x_{1}\right)}
$$

where $y_{0}$ is preselected constant, usually the value in a predetermined initial time. This rate does not satisfy Axiom A2, and hence there is no rational reason to use it. Due to this rate polynomials of the first degree

$$
f: t \mapsto y_{0} \cdot\left(1+\hat{\kappa}\left(x_{1}, y_{1}, x_{2}, y_{2}\right) \cdot t\right) .
$$

\section{Infinitesimal Version}

In macroeconomics an instantaneous measure of rate of growth is often needed. This may for a function $f$ be naturally given by a limiting process as (see (2))

$$
\begin{aligned}
v(f)\left(x_{0}\right) & =\lim _{x \rightarrow x_{0}} \kappa\left(x_{0}, f\left(x_{0}\right), x, f(x)\right) \\
& =\lim _{x \rightarrow x_{0}} \phi\left(\left(\frac{f(x)}{f\left(x_{0}\right)}\right)^{\left(\frac{1}{x-x_{0}}\right)}\right)=\phi\left(\mathrm{e}^{\frac{f^{\prime}\left(x_{0}\right)}{f\left(x_{0}\right)}}\right) .
\end{aligned}
$$

The number $v(f)\left(x_{0}\right)$ is called a $v$-rate of growth of f at point $x_{0}$. And for $\kappa=\kappa_{1}$ (see (4)) we have

$$
v_{1}(f)\left(x_{0}\right)=\mathrm{e}^{\frac{f^{\prime}\left(x_{0}\right)}{f\left(x_{0}\right)}}-1 .
$$

In macroeconomics a measure is used, denoted by $\tilde{v}$ obtained from (6) choosing $\phi=\ln$,

$$
\tilde{v}(f)\left(x_{0}\right)=\frac{f^{\prime}\left(x_{0}\right)}{f\left(x_{0}\right)} .
$$

In an analogous way we may use the same function for the rate of growth on interval $\left\langle x_{0}, x_{1}\right\rangle$ yielding

$$
\begin{aligned}
& \tilde{\kappa}\left(x_{0}, f\left(x_{0}\right), x_{1}, f\left(x_{1}\right)\right) \\
& =\ln \left(\left(\frac{f\left(x_{0}\right)}{f\left(x_{1}\right)}\right)^{\left(\frac{1}{x_{0}-x_{1}}\right)}\right)=\frac{\ln \left(f\left(x_{0}\right)\right)-\ln \left(f\left(x_{1}\right)\right)}{x_{0}-x_{1}}
\end{aligned}
$$

which represents the relative change of the composite function $\ln \circ f$ with respect to the change of the argument of the function. Notice, that the same limit has the simple interest $\hat{\kappa}$ (see (5)) letting $x_{1} \rightarrow x_{2}$.

\section{Consequence for the Interest Rate Calculations}

Using (2), the expression

$$
\kappa\left(x_{1}, f\left(x_{1}\right), x_{2}, f\left(x_{2}\right)\right)=\phi\left(\frac{f\left(x_{2}\right)}{f\left(x_{1}\right)}\right)^{\frac{1}{x_{2}-x_{1}}}
$$

is the rate of growth of function $f$ per unit of time. For 
instance, if $f$ represents how the state a dead account (neither deposits nor withdrawals) depends on time assuming $x_{1}, x_{2}$ are moments of time and the unit of time is a year, then $\kappa\left(x_{1}, f\left(x_{1}\right), x_{2}, f\left(x_{2}\right)\right)$ is the interest rate per a year, whereas if we choose in (2)

$$
\phi(x)=x^{t}-1 \text {, }
$$

we get (denoting the resulting function by $\kappa_{t}$ )

$$
\kappa_{t}\left(x_{1}, f\left(x_{1}\right), x_{2}, f\left(x_{2}\right)\right)=\left(\frac{f\left(x_{2}\right)}{f\left(x_{1}\right)}\right)^{\frac{1}{x_{2}-x_{1}}}-1
$$

which is a compound interest related to time segment $t=x_{2}-x_{1}$. Besides, it holds

$$
\kappa_{1}=\left(\frac{\kappa_{1}}{t}+1\right)^{t}-1 .
$$

It is known, that banks at the beginning of the past century (due to practical reasons stemming from the nonexistence of computers) used to find the value $\kappa_{1 / t}$ for small $1 / t$ the approximation by Taylor polynomial of the first degree of function (11) which gives the result

$$
\left(\kappa_{1}+1\right)^{\left(\frac{1}{t}\right)}-1 \cdot \frac{\kappa_{1}}{t}+O\left(\kappa_{1}^{2}\right)
$$

(where $O$ is Bachmann-Landau big-O). Consequently, supposing interest rate was known for some time interval (e.g. a month), the interest rate for shorter intervals (e.g. a day) was calculated dividing by 30 instead of as the 30th root. To legalize this inaccuracy, the notion of "an interval of adding of interests" was introduced with the clause, that if the current interval was shorter than that under assumption, the interest will be calculated multiplying only by a linear part of the increment of the interest rate. Hence function $f$ representing the state of account being in a steady state was changed from exponential to piecewise linear having with the original exponential curve common only breaking points. This practice is still surviving, despite banks use software that is definitely capable to calculate the roots. The reason rests (probably) with the shortage of management theoretical competence. The difference between the exact value and its approximation, i.e. an error of approximation is an increasing function when time approaches to infinity having finite limit $\left|e^{\kappa}-1-\kappa\right|$ because it holds

$$
\begin{aligned}
\lim _{t \rightarrow \infty}\left(1+\frac{\kappa}{t}\right)^{t}-1 & =\lim _{t \rightarrow \infty}\left(\left(1+\frac{\kappa}{t}\right)^{\frac{t}{\kappa}}\right)^{\kappa}-1 \\
& =\mathrm{e}^{\kappa}-1 .
\end{aligned}
$$

This limit is employed in a number of books on financial mathematics, although its interpretation is rather problematic. When we calculate compound interest and manipulate with a compound interest as with a simple interest in such a way that we divide time interval in equidistant subintervals and apply the interest that is the linear part of the approximation for these subintervals, we obtain the result, whose limit for the number of subintervals approaching to infinity is given by formula (12) A magic appearance of Euler constant in this calculation gave birth the notion of continuous compounding. It may be simply verified that it is in fact a compound interest, where in formula (4) the value $\ln \left(\kappa_{1}+1\right)$ instead of $\kappa_{1}$ is applied. The number $\ln \left(\kappa_{1}+1\right)$ may be obtained as a rate of growth when putting $\phi=\ln$ in (2) and then by limiting we get $\tilde{v}$ as in (8).

\section{Inverse Problem}

Let us use for the rate of growth formula (7) and denote $v_{1}=v$ with argument $t$ in the sequel. Then we have for a fixed $t_{0}$

$$
v(f)\left(t_{0}\right)=\mathrm{e}^{\frac{f^{\prime}\left(t_{0}\right)}{f\left(t_{0}\right)}}-1 .
$$

Supposing $f$ is given, then (13) is the formule to find the rate of growth $v$. Alternatively, when $v$ is given, then (13) is a differential equation to get the function $f$. This equation can be rearranged equivalently to

$$
\ln (v(t)+1)=(\ln \circ f)^{\prime}(t)
$$

or

$$
f^{\prime}(t)=\ln (v(t)+1) \cdot f(t)
$$

with the solution

$$
f(t)=\mathrm{e}^{\left(\int_{0}^{t} \ln (v(s)+1) \mathrm{d} s\right)} f(0),
$$

where $v(s)$ is the interest rate per unit of time at the moment $s$. Performing the same calculation for $\tilde{v}$ (see (8)), we get

$$
\tilde{v}(f)\left(t_{0}\right)=f^{\prime}\left(t_{0}\right) f\left(t_{0}\right)
$$

with the solution

$$
f(t)=\mathrm{e}^{\int_{0}^{t} v(s) \mathrm{d} s} f(0) .
$$

Although the formula (15) is clearly simplier than (14), it has disadvantage, because it yields quantitatively bad results. For instance if we substitute a constant interest rate in (15), we do not obtain the formula for a compound interest! The following example illustrates the use of formula (14).

Example. We assume that the inflation rate per a unit of time (e.g. a year) at time 0 and time 1 is known. Suppose that the inflation rate per unit of time at time 0 is 0.1 and 0.2 at time 1 . Deliberate on the inflation rate on interval $\langle 1,0\rangle$. It is evident that this depends on the 
changes of the inflation rate on $\langle 1,0\rangle$. Consider the following four cases of the inflation rate:

$$
\begin{aligned}
& \overline{\iota_{1}}(u)= \begin{cases}0.1, & \text { if } u<1 \\
0.2, & \text { if } u \geq 1,\end{cases} \\
& \overline{\iota_{2}}(u)=\frac{u^{2}}{10}+0.1, \\
& \overline{\bar{\zeta}_{3}}(u)=\frac{u}{10}+0.1, \\
& \bar{\iota}_{4}(u)= \begin{cases}0.1, & \text { if } u \leq 0 \\
0.2, & \text { if } u>0 .\end{cases}
\end{aligned}
$$

Notice that the first and the last cases are trivial - the rate is constant and the interval has a unit length and thus the inflation rate should be the same constant. The general formula must give the same result. By (14) we have

$$
f(1)=f(0) \cdot \mathrm{e}^{\left(\int_{0}^{1}\left(\ln \left(1+\bar{t}_{i}(u) \mathrm{d} u\right)\right)\right)}-1 .
$$

Applying (16) we get consecutively (setting $f(0)=1$ )

$$
\begin{aligned}
& \text { for } i=1: f(1)=\mathrm{e}^{\left(\int_{0}^{1} \ln (1.1) \mathrm{d} u\right)}-1=0.1 \\
& \text { for } i=2: f(1)=\mathrm{e}^{\left(\int_{0}^{1} \ln \left(1.1+u^{2} / 10\right) \mathrm{d} u\right)}-1=0.132945354 \cdots \\
& \text { for } i=3: f(1)=\mathrm{e}^{\left(\int_{0}^{1} \ln (1.1+u / 10) \mathrm{d} u\right)}-1=0.149637533 \cdots \\
& \text { for } i=4: f(1)=\mathrm{e}^{\left(\int_{0}^{1} \ln (1.2) \mathrm{d} u\right)}-1=0.2 .
\end{aligned}
$$

Now, applying (15) we obtain results

$$
\begin{aligned}
& \mathrm{e}^{\int_{0}^{1} 0.1 \mathrm{~d} u}-1=0.105170918 \\
& \mathrm{e}^{\int_{0}^{1} 1 / 10 u^{2}+0.1 \mathrm{~d} u}-1=0.142630812 \\
& \mathrm{e}^{\int_{0}^{1} 1 / 10 u+0.1 \mathrm{~d} u}-1=0.161834243 \\
& \mathrm{e}^{\int_{0}^{1} 0.2 \mathrm{~d} u}-1=0.221402758 .
\end{aligned}
$$

The results are surprisingly not equal (particularly the first and the last one) which is an evident failure. Formula for the future value of the compound interest in case of constant interest rate is given by

$$
f(t)=f(0) \cdot(1+\xi)^{t} .
$$

In case of piecewise constant interest rate, i.e. if $I_{i}$ are the values of constant interest rate per year on time intervals $\left(t_{i}, t_{i+1}\right), i=0, \cdots, n$, then the interest rate per $\bigcup_{i=0}^{n}\left(t_{i}, t_{i+1}\right\rangle$ is given by

$$
\prod_{i=0}^{n-1}\left(1+I_{i}\right)^{\left(t_{i+1}-t_{i}\right)} .
$$

\subsection{Theorem}

Formula (17) is a special case of formula (18) for a con- stant interest rate and formula (18) is a special case of formula (14) for a piecewise constant interest rate.

Proof: Assume $v(t)=I$ is constant. Then there holds

$$
\mathrm{e}^{\left(\int_{0}^{t} \ln (1+I) \mathrm{d} u\right)}=\mathrm{e}^{(t \cdot \ln (1+I))}=\mathrm{e}^{\left(\ln (1+I)^{t}\right)}=(1+I)^{t}
$$

and hence the first part of the statement holds true. Now let $v$ be piecewise constant possessing values $I_{i}$ on intervals $\left(t_{i}, t_{i+1}\right), i=0, \cdots, n$ and $\chi_{A}$ be a characteristic function of set $A$. We have $\imath(t)=\sum \chi_{\left(t_{i}, t_{i+1}\right)} \cdot I_{i}$ and hence

$$
\begin{aligned}
& \mathrm{e}^{\left(\int_{0}^{t} \ln \left(1+\chi_{\left(t_{i}, t_{i+1}\right)}\right) \cdot I_{i} \mathrm{~d} u\right)} \\
& =\mathrm{e}^{\left(\sum_{i=0}^{n-1}\left(t_{i+1} \ln \left(1+I_{i}\right)\right)-t_{i} \ln \left(1+I_{i}\right)\right)}=\prod_{i=0}^{n-1} \mathrm{e}^{\left(t_{i+1}-t_{i}\right) \cdot \ln \left(1+I_{i}\right)} \\
& =\prod_{i=0}^{n-1} \mathrm{e}^{\ln \left(1+I_{i}\right)^{\left(t_{i+1}-t_{i}\right)}}=\prod_{i=0}^{n-1}\left(1+I_{i}\right)^{t_{i+1}-t_{i}}
\end{aligned}
$$

and the proof is completed.

\subsection{Theorem}

Formula (14) is a limit case of formula (17).

Proof: First we show, that for every continuous function $f$ defined on a closed interval, there exists a sequence of piecewise constant functions $\xi_{i}$ with the property $\xi_{i} \rightarrow f$. Let $f$ be a continuous function. Due to the assumption $\operatorname{Dom}(f)$ is the compact set. Let $\epsilon$ be positive real number. For every $x \in \operatorname{Dom}(f)$ we find a neighborhood $O(x)$ such that $f(O(x)) \subset O_{\epsilon / 2}(f(x))$. $\{O(x)\}_{x \in \operatorname{Dom}(f)}$ forms a covering of $\operatorname{Dom}(f)$. Choose a finite subcovery $\Omega$ and define $\delta=\min _{U \in \Omega}(\operatorname{Diam}(U))$, where $\operatorname{Diam}(U)$ is a diameter of $U$. Consider a partition of $\operatorname{Dom}(f)$ given by $n$ disjoint subintervals $\left(J_{i}^{\epsilon}\right)_{i}^{n}$ of the length $\delta$. In every subinterval $J_{i}^{\epsilon}$ we choose a point $x_{i}$, and denote $y_{i}=f\left(x_{i}\right)$. Further, define $\zeta_{\epsilon}(x)=y_{i}$ for all $x \in J_{i}^{\epsilon}$. Then for every $x \in \operatorname{Dom}(f)$ it holds $\left|f(x)-\zeta_{\epsilon}(x)\right| \leq \epsilon$ and for $\xi_{n}=\zeta_{\frac{1}{2^{n}}}$ the above property is satisfied. Since the functional

$$
\Phi: \iota \mapsto \mathrm{e}^{\int_{0}^{t} \ln (1+l(s)) \mathrm{d} s}
$$

is continuous in the topology of uniform convergence, we get

$$
\lim _{i \rightarrow \infty} \Phi\left(\psi_{i}\right)=\Phi\left(\lim _{i \rightarrow \infty} \psi_{i}\right)=\Phi(\Psi)
$$

and the proof is completed.

\section{Interest Rate of Simple Compounding}

As an impact of the preceding considerations let us point to the issue of simple compouding. Simple compounding 
is a situation in which dependence of a quantity on time is a polynomial of the first degree (let us call the dependence of the quantity on time a state function). In this situation, special rate of growth is used (see (5)) but this rate has fundamental conceptual flaws. One of them rests with the mixing of different ways of measuring the rate of growth.

From the above considerations we can conclude, that in all situations the only one rate of growth is sufficient given by (2). In what follows we compute the rate of growth of a quantity, which is simply compounded (it may be called "compound interest rate of a simple compounding").

It is evident if the rate of growth function is constant and positive, then the state function is increasing and convex. Further if the rate of growth function is positive and decreases sufficiently quickly, then the state function is increasing but concave. Now we are looking for the rate of growth function, which makes the state function affine, i.e. it has the form of a polynomial of the first degree. To find it, we consider the state function (see (14))

$$
t \mapsto f(0) \mathrm{e}^{\int_{0}^{t} \ln (1+\xi(s)) \mathrm{d} s} .
$$

Its derivative is given by

$$
t \mapsto f(0) \ln (1+\xi(t)) \mathrm{e}^{\int_{0}^{t} \ln (1+\xi(s)) \mathrm{d} s}
$$

and second derivative by

$$
\begin{aligned}
t \mapsto f(0) \mathrm{e}^{\int_{0}^{t} \ln (1+\xi(s)) \mathrm{d} s} & \\
& \cdot \frac{\left(\xi^{\prime}(t)+(\ln (1+\xi(t)))^{2}+(\ln (1+\xi(t)))^{2} \xi(t)\right)}{1+\xi(t)} .
\end{aligned}
$$

Since the state function is polynomial of the first degree its second derivative must be equal to zero. If $f(0) \neq 0$ and $\xi(t)>0$, then second derivative is equal to zero for such $\xi(t)$, that are solution of the differential equation

$$
\xi^{\prime}(t)+(\ln (1+\xi(t)))^{2}+(\ln (1+\xi(t)))^{2} \xi(t)=0 .
$$

The solution of (20) is

$$
\xi(t)=\mathrm{e}^{\left(\frac{1}{t-C}\right)}-1
$$

for any constant $C$. For the given value $\xi(0)$ we get from (21)

$$
C=-\frac{1}{\ln (1+\xi(0))}
$$

and hence the partial solution is

$$
\begin{aligned}
\xi(t) & =\mathrm{e}^{\left(\frac{1}{t+\frac{1}{\ln (1+\xi(0))}}\right)}-1 \\
& =(1+\xi(0))\left(\frac{1}{t \ln (1+\xi(0))+1}\right)-1 .
\end{aligned}
$$

If we substitute this rate into (14), we get

$$
\begin{aligned}
f(t) & =f(0) \mathrm{e}^{\left(\int_{0}^{t} \ln \left((1+\xi(0))\left(\frac{1}{s \ln (1+\xi(0))+1}\right)\right) \mathrm{d} s\right)} \\
& =f(0)(t \ln (1+\xi(0))+1)
\end{aligned}
$$

which is really an affine function, i.e. a state function of a simple compounding. Applying (5), substituting $x_{1}$ and $x_{2}$ arbitrary and setting coresponding $y_{1}=f\left(x_{1}\right)$, $y_{2}=f\left(x_{2}\right)$ and $y_{0}=f(0)$ due to (23), we obtain formula for the rate of simple compounding of (23)

$$
\iota=\ln (1+\xi(0)) \text {. }
$$

while rate of growth function of (23) is

$$
\mathrm{e}^{\frac{l}{t \cdot l+1}}-1
$$

\section{Conclusions}

In the article we presented an explicit formula for all possible rates of growth possessing natural properties (described by Axioms A1-A4) (see (2)). Further we derived the new formula for the rate of growth at a point by limiting process. This formula enables to assign to state function its rate of growth (see (7)). Moreover formula is given to find a state function on condition its rate of growth function at any point is known (see (14)).

Although the choice of Axioms A1-A4 seems to be natural, the condition that any exponential function is a steady state function is of crucial importance. It is an open problem of finding a simpler condition or to show that this condition may be derived from the axioms.

\section{REFERENCES ${ }^{1}$}

[1] R. J. Barro and X. Sala-i-Martin, "Economic Growth," 2nd Edition, 2003.

[2] J. Dupačová, J. Hurt and J. Št epán, "Stochastic Modeling in economics and Finance," Kluwert Academic Publishers, 2002.

[3] J. Aczél, "Lectures on Functional Equations and Their Applications," Academic Press, New York, 1966.

[4] V. Studený, "Functional Equation of the Rate of Inflation," e-Print Archive of Coronell University, 2003.

\footnotetext{
${ }^{1}$ The first usage of the new way and new formulas can reader find in [4] The usual approach to the problem - problematic, as shown in this article - can be seen in [5-8]. For more details of mathematical analysis used here see [9].
} 
http://arxiv.org/abs/math/0307395

[5] J. C. Van Horne, "Financial Markets, Rates and Flows," Prentice Hall, Englewood Clifs, 1978.

[6] R. C. Merton, “Continuous Time Finance," Blackwell, Cambridge, 1992.

[7] I. Karatzas and S. Shreve, "Methods of Mathematical
Finance," Springer, New York, 1998.

[8] T. Cipra, "Mathematics of Securities," HZ, Praha, 2000.

[9] J. Dieudonné, "Treatise on Analysis, Vol. III, Pure and Applied Mathematics, Vol. 10-III," Academic Press, New York, London, 1972.

\section{Classification Codes:}

\section{MSC (Mathematics Subject Classification)}

26A12, Rate of growth of functions, orders of infinity, slowly varying functions

62P20, Applications to economics

39B22, Equations for real functions

91B02, Fundamental topics (basic mathematics, methodology; applicable to economics in general)

91B28, Finance, portfolios, investment

91B24, Price theory and market structure

34A05, Explicit solutions and reductions

\section{JEL (Journal of Economic Literature)}

E4, Money and Interest Rates: General (includes measurement and data)

E43, Determination of Interest Rates; Term Structure of Interest Rates

$\mathrm{C} 02$, Mathematical Methods

C43, Index Numbers and Aggregation

C63, Computational Techniques

C65, Miscellaneous Mathematical Tools 